\title{
SCIDiC
}

\author{
International Journal of Dentistry and Oral Science (IJDOS) \\ ISSN: 2377-8075
}

\section{Evaluation of Antimicrobial Activity of $\beta$-tricalcium Phosphate/Calcium Sulfate Mixed-up with Gentamicin: In-Vitro Study}

Research Article

Khaled Jamal Zain ${ }^{1}$, Bassel Adnan Brad², Saleh Bashar Al Kurdi³ ${ }^{3}$ Mohamad Mhd Ghyath Jumaa ${ }^{4}$, Muaaz Alkhouli ${ }^{* *}$

${ }^{1}$ MSc Resident, Department of Oral and Maxillofacial Surgery, Faculty of Dentistry, Damascus University, Damascus, Syria.

${ }^{2}$ Associate Professor, head of Department Oral and Maxillofacial Surgery, Faculty of Dentistry, Damascus University, Damascus, Syria.

${ }^{3} \mathrm{PhD}$ Resident, Department of Pediatric Dentistry, Faculty of Dentistry, Damascus University, Damascus, Syria.

${ }^{4} \mathrm{BSc}$ in Pharmacy, Arab International University, Damascus, Syria.

${ }^{5}$ PhD Candidate, Department of Pediatric Dentistry, Faculty of Dentistry, Damascus University, Damascus, Syria.

Abstract

Objectives: An in-vitro study was designed to investigate the antimicrobial activity of $\beta$-tricalcium phosphate/calcium sulfate in comparison to Xenograft, blended with either gentamicin or saline.

Methods and materials: Employing Kerby-Bauer disk diffusion test,twelve Petri dishes $(90 \mathrm{~mm})$ with two models of culture mediums were used to cultivate three bacterial strains: Muller-Hinton Agar (for P. Aeruginosa/S. Aureus)andSheep Blood Agar (for Enterococcus faecalis).Then, studied materials were divided into four groups (Bovine, Bovine with gentamicin, EthOss and EthOss with gentamicin), mixed-upand transmittedinto their holes in each dish. After 24h of incubation, inhibition zones were noted and measured bya digital caliper.In the end statistical analysis were completed with One Way ANOVA followed by Tuckey HSD test on SPSS17.

Results: One Way ANOVA and Tuckey HSD remarked astatically significant difference between all pairs for Enterococcus faecalis $(\mathrm{p}<0.05)$, except of Bovine/ EthOss pair. When it comes up to Staphylococcus aureus, a significant difference was observed between all pairs $(\mathrm{p}<0.05)$, except Bovine/ EthOss and EthOss Gentamicin/ Bovine Gentamicin pairs, both were effective In bacterial elimination. Finally, only Bovine Gentamicin was functional with Pseudomonas aeruginosa $(p<0.05)$. Conclusion: $\beta$-tricalcium phosphate/calcium sulfate and Xenograft antibacterial abilities were improved when mixed with Gentamicin. Xenograft was preferable as an antibiotic carrier when it comes to Pseudomonas aeruginosa.

Keywords: $\beta$-tricalcium Phosphate; Calcium Sulfate; Xenograft; Gentamicin; Antimicrobial.

\section{Introduction}

Dental implantation is a common treatment for tooth loss, despite survival rates of $96.33 \%$ after 8 years of the procedure, biological complications are likely to occur, including peri-implantitis, which includes bone loss and peri-implant mucositis.[1]

The principle goal of peri-implantitis treatment is to manage theinfection, prevent further bone resorption, maintain aesthetics and enhance bone regeneration in the area of bone loss.[2]

Various types of bone grafts have been used for bone regeneration. Bone grafts are classified according to their source to: Autogenous Bone Grafts (tibia, Iliac, ramus), Allograft (FDBA,
DFDBA), Xenograft (Bovine) and Alloplastic Bone Graft (Calcium Sulfate, Calcium Phosphate).[3]

Peri-implantitis etiology is similar to periodontitis. In both of themgerms attach to the implant`s or tooth`s surface causing bone resorption.[2]

The main goal of treatment is the removal of bacterial plaque.[2] Pseudomonas aeruginosa(a Gram-negative, facultative anaerobic rod bacterium), Staphylococcus aureus (Gram-positive, facultative aerobe, round-shaped, bacterium) and Enterococcus faecalis ( Gram-positive, facultative anaerobe) were frequent found in peri implantitis.In addition to that, they were accusedwith implant failure. $[4,5]$

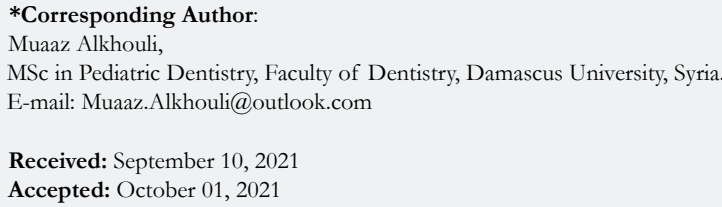

Copyright: Muaaz Alkhouli ${ }^{\circ} 2021$. This is an open-access article distributed under the terms of the Creative Commons Attribution License, which permits unrestricted use, distribution and reproduction in any medium, provided the original author and source are credited. 
Some researchers have suggested adding antibiotic to the bone graft that used for regenerative therapy around Implants to reduce the bacterial Load. [6]

The benefits of local release is the ability to provide high local antibiotic concentrations without systemic toxicity. It has been suggested that such high concentrations can even penetrate a biofilm. $[7,8]$

One of the most used material is Polymethylmethacrylate (PMMA). This material is an acrylic non-absorbable material that must be removed in a surgical procedure. PMMA beads may continue to excrete low levels of antibiotic even up to five years, which may generate multi-drug resistant organisms. Furthermore, once the antibiotic levels are too low to kill organisms the PMMA itself can become colonized. $[9,10]$

Calcium sulfate bone graft is an absorbable material does not need a surgical procedure to remove, several studies have been aimed to combination antibiotic with calcium sulfate substitutes depending on it biocompatibility, porosity, and biodegradability. [10]

Gentamicin is anaminoglycoside, which is used for treatment of serious infections caused byaerobic gram-negative bacilli such as Pseudomonas aeruginosa.It has a limited effect on gram-positive germs like staphylococcus aureus and enterococcus faecalis.Yet, Gentamicin's clinical utility is limited due to its serious toxicity. [11] Coating titanium implants withGentamicin found no negative impact on osteoblast function.[12]

The aim of this study is to evaluate the efficacy of the biphasic absorbable bone graft (35\% calcium sulfate $/ 65 \% \beta$-tricalcium phosphate) mixed with either normal saline or gentamicin $2 \mathrm{mg} /$ $\mathrm{ml}$, in comparison to bovine bone graft mixed with eithernormal saline or gentamicin $2 \mathrm{mg} / \mathrm{mlin}$ inhibitingP.aeruginosa,S.aureusand E.faecalis growth.

\section{Methods and Materials}

Three clinical bacterial strains isolates were collected from different patients who were administrated to Al-Mowasat Hospital- apartment of Bacteriology tests Laboratory. The study was accomplished with diffusion test method. Twelve Petridishes (90 $\mathrm{mm}$ ) with two models of bacterial culture mediums were used (Muller-Hinton Agar for P. Aeruginosa and S. Aureus / Blood Agar for Enterococcus faecalis isolated from necrotic root canals). To perform Kirby Bauer disk, bacterial density was controlled by PhonexSpec at $0.5 \mathrm{McF}$ arland Standard (1.5x $108 \mathrm{CFU} / \mathrm{ml})$. Then a sterile swabs was dipped into inoculums tube, to remove the ex- cess fluid the swab was pressured around the tube walls. Bacteria was inoculated over its specified agar dish, then was left at $37^{\circ}$ for 20 minutes to dry.

At the margins of each agar dish a hole of $(4 \mathrm{~mm})$ depth and $(6 \mathrm{~mm})$ diameter was punched; the diameter was defined to simulate the standard diameter of the antibiotic sensitivity disk. Four mixed materials were prepared:

1. Biphasic absorbable alloplastic bone substitute (EthOss ${ }^{\circledR}$, EthOss Regeneration Ltd., Silsden, UK) which consists of $\beta$ Tricalcium Phosphate (65\%) and Calcium Sulfate (35\%) mixed with normal saline.

2. EthOss mixed with Gentamicin $2 \mathrm{mg} / \mathrm{ml}$ (Gentacine ${ }^{\circledR}$, Ibn Hayyan Pharma, Homs, Syria).

3. Bovine bone graft (MedPark Bone-D ${ }^{\circledR}$, MedPark, Busan, Korea) mixed with normal saline.

4. MedPark Bone-D mixed with Gentamicin $2 \mathrm{mg} / \mathrm{ml}$.

Then each mix was freshly transformed to fill its hole in each agar dish, then we kept it for one hour at room temperature to insure the expansion of the materials through the Agar. Thereafter, they were incubated at $37^{\circ} \mathrm{C}$ for 24 hours before inducting the test.

Inhibition zones surround each hole were measured by the digital caliper, to observed the anti-bacterial activity (higher inhibition zone diameters) of the tested mixture.

Finally, SPSS program was used to accomplish the statistical descriptive and analytic processes. At significance level of 0.05 , multiple comparison between groups was done using One Way ANOVA test followed by Tuckey HSD.

\section{Results}

Statistical analysis were performed with SPSS software. Means, standard deviations, minimum and maximum values of each tested material according to its efficacy in inhibiting microbial growth of tested microorganisms is described in table 1.

Shapiro-Wilk normality test stated a normal distribution of the values. Therefore, One Way ANOVA test was carried out, it conducted a significant difference for all studied materials against each studied microorganisms $(\mathrm{p}<0.05)$ table1.

To compare between material pairs Tuckey HSD test was accomplished. A significant difference was seen between all pairs for Enterococcus faecalis $(\mathrm{p}<0.05)$, except Bovine/EthOss pair. For Staphylococcus aureus, a significant difference was observed between all pairs $(\mathrm{p}<0.05)$, except Bovine/EthOss and EthOss

Figure 1: a. b. measure the diameter of inhibition zone by digital caliper.

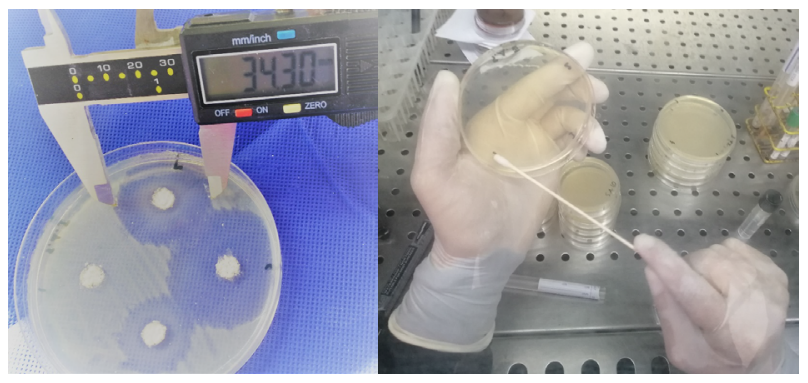


Table 1.

\begin{tabular}{|c|c|c|c|c|c|c|}
\hline Microorganisms & Tested material & Mean & Std. Deviation & Minimum & Maximum & ANOVA Sig. \\
\hline \multirow{4}{*}{ Enterococcus faecalis } & Bovine & 0 & 0 & 0 & 0 & 0 \\
\cline { 2 - 7 } & EthOss & 0 & 0 & 0 & 31.2 \\
\cline { 2 - 7 } & Bovine Gentamicin & 30.565 & 0.575 & 29.9 & 27.6 & 0 \\
\cline { 2 - 7 } & EthOss Gentamicin & 27.307 & 0.229 & 27.1 & 0 & 26.2 \\
\hline \multirow{5}{*}{ Staphylococcus aureus } & Bovine & 0 & 0 & 10.7 & 41.3 \\
\cline { 2 - 7 } & EthOss & 18.178 & 8.116 & 36.2 & 34.8 & 0 \\
\cline { 2 - 7 } & Bovine Gentamicin & 37.715 & 2.408 & 34.2 & 0 \\
\cline { 2 - 7 } & EthOss Gentamicin & 34.51 & 0.336 & 0 & 0 & 0 \\
\cline { 2 - 7 } & Bovine & 0 & 0 & 1.223 & 19.4 & 22.3 \\
\cline { 2 - 7 } & Bseudomonas aeruginosa & 20.838 & 0 & 0 & 0 \\
\cline { 2 - 6 } & Eovine Gentamicin & 0 & 0 & 0 & 0 \\
\cline { 2 - 6 }
\end{tabular}

Table 2.

\begin{tabular}{|c|c|c|c|}
\hline $\begin{array}{c}\text { Multiple Comparisons } \\
\text { Tukey HSD }\end{array}$ & & & \\
\hline Dependent Variable & (I) Group & (J) Group & Sig. \\
\hline \multirow{6}{*}{ Enterococcus faecalis } & \multirow{3}{*}{ Bovine } & EthOss & 1.000 \\
\hline & & Bovine Gentamicin & .000 \\
\hline & & EthOss Gentamicin & .000 \\
\hline & \multirow{2}{*}{ EthOss } & Bovine Gentamicin & .000 \\
\hline & & EthOss Gentamicin & .000 \\
\hline & Bovine Gentamicin & EthOss Gentamicin & .000 \\
\hline \multirow{6}{*}{ Staphylococcus aureus } & \multirow{3}{*}{ Bovine } & EthOss & 1.000 \\
\hline & & Bovine Gentamicin & .000 \\
\hline & & EthOss Gentamicin & .000 \\
\hline & \multirow{2}{*}{ EthOss } & Bovine Gentamicin & .000 \\
\hline & & EthOss Gentamicin & .001 \\
\hline & Bovine Gentamicin & EthOss Gentamicin & .713 \\
\hline \multirow{6}{*}{ Pseudomonas aeruginosa } & \multirow{3}{*}{ Bovine } & EthOss & 1.000 \\
\hline & & Bovine Gentamicin & .000 \\
\hline & & EthOss Gentamicin & 1.000 \\
\hline & \multirow{2}{*}{ EthOss } & Bovine Gentamicin & .000 \\
\hline & & EthOss Gentamicin & 1.000 \\
\hline & Bovine Gentamicin & EthOss Gentamicin & .000 \\
\hline
\end{tabular}

Gentamicin/Bovine Gentamicin pairs. Finally, only Bovine Gentamicin revealed a significant difference with all compared materials for Pseudomonas aeruginosa $(\mathrm{p}<0.05)$ table 2 .

\section{Discussion}

Successful perimplantitis treatment requires effective elimination of persistent bacterial population from implantation region.[13] This is carried out through mechanical and chemical treatment of implant surface.[14] In addition to that, bone graft might possess some antimicrobial activity, driving the managed perimplantitis area to promising long-term outcomes.

Staphylococcus aureus, Enterococcus faecalis and Pseudomonas aeruginosa were tested as long as they frequently exist in implantrelated bone infections. $[15,16]$

Agar diffusion method is recognized as standardized methodto determine primary antimicrobial capacity of the studied materials. However, it has some limitation such as its inability in recognizing whether the studied material has bactericidal or bacteriostatic effects, therefore those results must be read with caution.

\section{Group 1 (Enterococcus faecalis):}

Both bovine and EthOss beads did not have any antimicrobial activity. Bovine is chemically inactive substance without any antimicrobial ability. When it comes to $\beta$-tricalcium phosphate/calcium 
Figure 2. a. The four materials aftertransformedto its holesin blood agar. b. The inhibition zone after $24 \mathrm{~h}$.

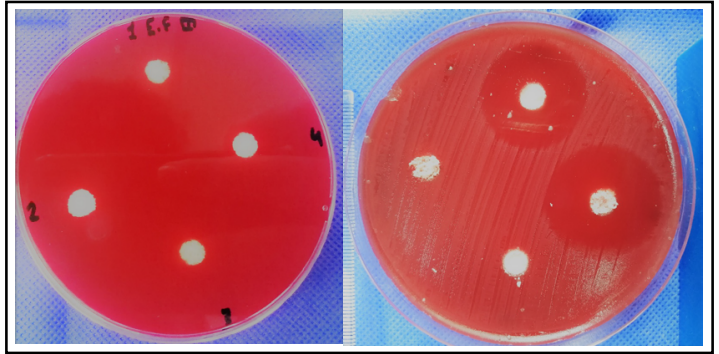

Figure 3. a. The four materials aftertransformed to its holes in Muller-hinton agar. b. The inhibition zone after $24 \mathrm{~h}$.

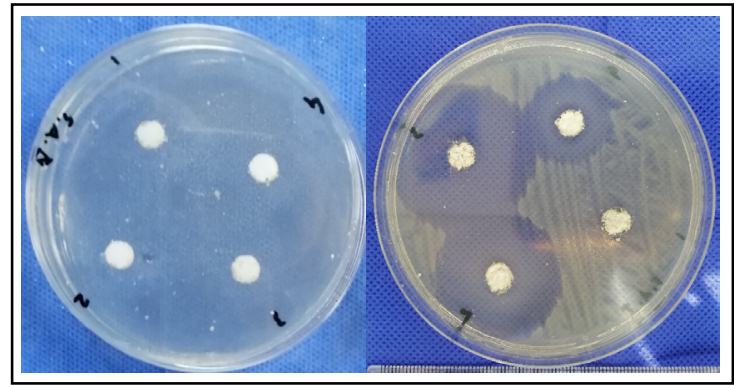

Figure 4. a. The four materials aftertransformed to its holes in Muller-hinton agar. b. The inhibition zone after $24 \mathrm{~h}$.

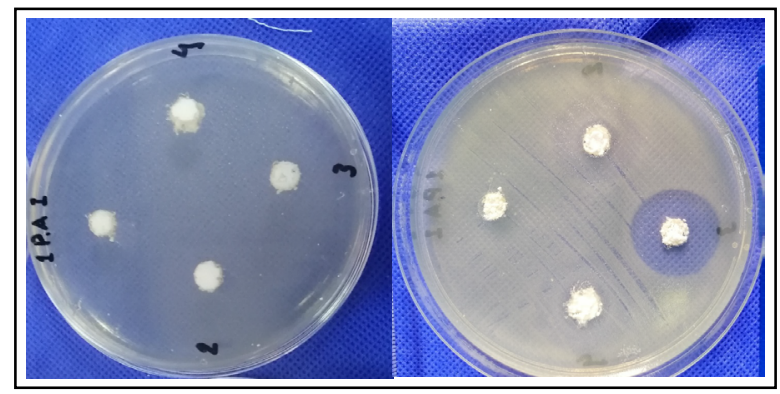

sulfate, there was no previous studies on its antimicrobial activity for E.faecalis.

On the other hand, mixing the studied bone-grafts with gentamicin showed a significant antimicrobial action; it seems that they acted as an antibiotic carrier helping in eliminationof bacterial load. However, it was obviousthat bovine with gentamicin had a slightly larger inhibition zone than EthOss with gentamicin (30.56 $\pm 0.57 \mathrm{~mm}, 27.30 \pm 0.22 \mathrm{~mm}$ ); it can be explained by the reduction of gentamicin's efficacy at acidic media [17], it was mentioned by Firgusson et al. thatEthOsstransforms from neutral to an acidic media at dissolve-stage. [6]

\section{Group 2 (Staphylococcus aureus):}

Bovine beads did not have any antimicrobial activity. However, EthOss demonstrated significant antimicrobial activity with an inhibition zone of $18.17 \pm 8.11 \mathrm{~mm}$ diameter.Shizou et al studied calcium phosphate against S.aureus implanted in Muller-Hinton agar, they conducted that an inhibition zone of $32.2 \pm 2.5 \mathrm{~mm}$ diameter was formed. [18]

Adding gentamicin to the studied bone-grafts displayed a significant antimicrobial action.

Bovine with gentamicin had a slightly larger inhibition zone than
EthOss with gentamicin $(37.71 \pm 2.40 \mathrm{~mm}, 34.51 \pm 0.33 \mathrm{~mm})$, without any statistical difference between them.

\section{Group 3 (Pseudomonas aeruginosa):}

When it comes to P.aeruginosa inhibition abilities; bovine, Eth Oss and EthOss with gentamicin antimicrobial activities were none. The acidic microenvironment produced by EthOss at dissolve stage may have affected gentamicin's efficacy. [17] Gentamicin's concentration after being mixed with EthOss seems to be lower than the Minimum Inhibitory Concentration (MIC) needed to kill P.aeruginosa. Which requires more investigation in further studies.

Bovine with gentamicin showed an inhibition zone of $20.83 \pm$ $1.22 \mathrm{~mm}$ diameter, it played the role of a physical carrier for gentamicin without any interactions in-between.

In present work, EthOss with gentamicin exhibited antimicrobial activity against all previous strains except P.aeruginosa. On the contrary, Bovine with gentamicin was effective on all strains so it might be better in immediate implantation cases that have a periapical lesion or in apicoectomy procedures.

EthOss solitarily or with gentamicin can be used in perimplantitis surgical regenerative interventions, due to its antimicrobial effect 
on S.aureus, this strain is frequently associated with implant-related bone infections [16].

Agar diffusion test standards of inhibition zone for Gentamicinmeasures: $19-27 \mathrm{~mm}$ for S.aureus, $16-22 \mathrm{~mm}$ for P.aeruginosa, and 15-25 mm for E. faecalis. [21, 22]

When it comes to E.faecalis group, inhibition zones diameter of Bovine with gentamicin and EthOss with gentamicin were 30.56 $\pm 0.57 \mathrm{~mm}$ and $27.30 \pm 0.22 \mathrm{~mm}$ respectively. Moreover, S.aureus group diameters for EthOss, Bovine with gentamicin and EthOss with gentamicin were $18.17 \pm 8.11,37.71 \pm 2.40 \mathrm{~mm}$ and 34.51 $\pm 0.33 \mathrm{~mm}$ respectively. Considering these results, andcomparing them to the critical values of gentamicin, it can be concluded that studied bone grafts preserve the antimicrobial action of gentamicin playing the role of appropriate medication carrier to the cured area.On the other hand, in P.aeruginosa group only Bovine with gentamicin had an inhibition zone with diameter of $20.83 \pm$ 1.22 , indicating an antimicrobial effect in comparison with critical gentamicin values.

Smaller inhibition zone diameter of EthOss with gentamicin compared to Bovine with gentamicin might be referred to the ingress of gentamicin solution into the composition of EthOss while it hardens, decreasing the initial concentration of the medicament.[6]

\section{Conclusion}

Last but not least, this in-vitro study reported that mixing $\beta$-tricalcium phosphate/calcium sulfate, and Xenograft with Gentamicin enhanced favorable antibacterial abilities to manage infected bone zones. Additionally, Xenograft was preferable as an antibiotic carrier especially when it comes to Pseudomonas aeruginosa. Finally, $\beta$-tricalcium phosphate/calcium sulfate bone grafts appears to have minimal antimicrobial activity against staphylococcus aureus.

\section{References}

[1]. Jang HW, Kang JK, Lee K, Lee YS, Park PK. A retrospective study on related factors affecting the survival rate of dental implants. J Adv Prosthodont. 2011 Dec 1;3(4):204-15.

[2]. Schwarz F, Derks J, Monje A, Wang HL. Peri-implantitis. Journal of clinical periodontology. 2018 Jun; 89 Suppl 1: S267-S290.

[3]. Laurencin C, Khan Y, El-Amin SF. Bone graft substitutes. Expert Rev Med Devices. 2006 Jan 1;3(1):49-57.

[4]. Lafaurie GI, Sabogal MA, Castillo DM, Rincón MV, Gómez LA, Lesmes YA, et al. Microbiome and microbial biofilm profiles of peri-implantitis: a systematic review. J. Periodontol. 2017 Oct;88(10):1066-89.

[5]. Flanagan D. Enterococcus faecalis and dental implants. J Oral Implantol. $2017 \mathrm{Feb} ; 43(1): 8-11$

[6]. Ferguson J, Diefenbeck M, McNally M. Ceramic Biocomposites as Biode- gradable Antibiotic Carriers in the Treatment of Bone Infections. J Bone Jt Infect. 2017 Jan 1;2(1):38-51.Pubmed PMID: 28529863.

[7]. Cabrita HB, Croci AT, Camargo OP, Lima AL. Prospective study of the treatment of infected hip arthroplasties with or without the use of an antibiotic-loaded cement spacer. Clinics. 2007;62:99-108.

[8]. Aiken SS, Cooper JJ, Florance H, Robinson MT, Michell S. Local release of antibiotics for surgical site infection management using high-purity calcium sulfate: an in vitro elution study. Surg Infect (Larchmt). 2015 Feb;16(1):5461.Pubmed PMID: 25148101.

[9]. Gitelis S, Brebach GT. The treatment of chronic osteomyelitis with a biodegradable antibiotic-impregnated implant. J Orthop Surg. 2002 Jun;10(1):53-60.

[10]. Butini ME, Cabric $S$, Trampuz A, Di Luca M. In vitro anti-biofilm activity of a biphasic gentamicin-loaded calcium sulfate/hydroxyapatite bone graft substitute. Colloids Surf B Biointerfaces. 2018 Jan 1;161:252-260.Pubmed PMID: 29096369.

[11]. Whalen, K., Lippincott ${ }^{\circ}$ Illustrated Reviews: Pharmacology. 2018: Wolters kluwer india Pvt Ltd.

[12]. Vester H, Wildemann B, Schmidmaier G, Stöckle U, Lucke M. Gentamycin delivered from a PDLLA coating of metallic implants: In vivo and in vitro characterisation for local prophylaxis of implant-related osteomyelitis. Injury. 2010 Oct;41(10):1053-9.Pubmed PMID: 20541756.

[13]. El Chaar E, Almogahwi M, Abdalkader K, Alshehri A, Cruz S. Decontamination of the Infected Implant Surface: A Scanning Electron Microscope Study. Int J Periodontics Restorative Dent. 2020 May 1;40(3):395-401.

[14]. Mellado-Valero A, Buitrago-Vera P, Solá-Ruiz MF, Ferrer-García JC. Decontamination of dental implant surface in peri-implantitis treatment: a literature review. Medicina oral, patologia oral y cirugia bucal. 2013 Nov; 18(6):e869-76.

[15]. Lewis CS, Supronowicz PR, Zhukauskas RM, Gill E, Cobb RR. Local antibiotic delivery with demineralized bone matrix. Cell Tissue Bank. 2012 Mar;13(1):119-27.

[16]. Ramanauskaite A, Daugela P, Juodzbalys G. Treatment of peri-implantitis: Meta-analysis of findings in a systematic literature review and novel protocol proposal. Quintessence Int. 2016;47(5):379-93.Pubmed PMID: 26574611.

[17]. Baudoux P, Bles N, Lemaire S, Mingeot-Leclercq MP, Tulkens PM, Van Bambeke F. Combined effect of $\mathrm{pH}$ and concentration on the activities of gentamicin and oxacillin against Staphylococcus aureus in pharmacodynamic models of extracellular and intracellular infections. J Antimicrob Chemother. 2007 Feb;59(2):246-53.Pubmed PMID: 17220162.

[18]. Wu S, Lei L, Bao C, Liu J, Weir MD, Ren K, et al. An injectable and antibacterial calcium phosphate scaffold inhibiting Staphylococcus aureus and supporting stem cells for bone regeneration. Mater Sci Eng C Mater Biol Appl. 2021 Jan;120:111688.Pubmed PMID: 33545850.

[19]. Zan R, Kutlu G, Hubbezoglu I, Sumer Z, Tunc T, Mutlu Z. Bactericidal effects of various irrigation solutions against staphylococcus aureus in human root canal. J Istanb Univ Fac Dent. 2015 Jan 31;49(1):19-26.Pubmed PMID: 28955521.

[20]. Woolfrey BF, Fox JM, Quall CO, Lally RT. Error rates associated with the use of recently proposed breakpoints for testing Pseudomonas aeruginosa versus gentamicin, tobramycin, and amikacin by the standardized disk agar diffusion test. Antimicrob Agents Chemother. 1983 Nov;24(5):764-70.Pubmed PMID: 6419674.

[21]. Hsueh PR, Ko WC, Wu JJ, Lu JJ, Wang FD, Wu HY, et al. Consensus statement on the adherence to Clinical and Laboratory Standards Institute (CLSI) Antimicrobial Susceptibility Testing Guidelines (CLSI-2010 and CLSI-2010-update) for Enterobacteriaceae in clinical microbiology laboratories in Taiwan. J Microbiol Immunol Infect. 2010 Oct;43(5):452-5.Pubmed PMID: 21075714.

[22]. Kronvall GÖ, Ringertz SI, NystrÖM SI, Rylander MA, Theodorsson EL. Comparison of $30 \mu \mathrm{g}$ and $120 \mu \mathrm{g}$ gentamicin disks for the prediction of gentamicin resistance in Enterococcus faecalis. APMIS. 1991 Jul;99(7-12):88792. 